\title{
Classifying Annihilating-Ideal Graphs of Commutative Artinian Rings
}

\author{
Amanda R. Curtis
}

Alexander J. Diesl

January 22, 2018

\begin{abstract}
In this article we investigate the annihilating-ideal graph of a commutative ring, introduced by Behboodi and Rakeei in [BR11a]. Our main goal is to determine which algebraic properties of a ring are reflected in its annihilating-ideal graph. We prove that, for artinian rings, the annihilating-ideal graph can be used to determine whether the ring in question is a PIR or, more generally, if it is a dual ring. Moreover, with one trivial exception, the annihilating-ideal graph can distinguish between PIRs with different ideal lattices. In addition, we explore new techniques for classifying small annihilating-ideal graphs. Consequently, we completely determine the graphs with 6 or fewer vertices which can be realized as the annihilating-ideal graph of a commutative ring.
\end{abstract}

\section{Introduction}

The idea of the zero-divisor graph of a ring is due to Beck [Bec88], in which the author is primarily concerned with colorings. In [AL99], Anderson and Livingston defined the zerodivisor graph of a commutative ring $R$, denoted $\Gamma(R)$, to be the graph whose vertices are the nonzero zero-divisors of $R$, and in which $x$ and $y$ are connected by an edge if $x y=0$. Since then, there have been many papers written on the subject of zero-divisor graphs and and their variants (of which there are many). Much of the work in this area can be loosely organized around two major motivating questions, which we will informally refer to as the "realization problem" and the "isomorphism problem".

The "realization problem" is the problem of determining which graphs can arise as zerodivisor graphs of rings. Implicit in this is the study of graph-theoretic properties (e.g. diameter, girth, clique number, genus, etc.) of zero-divisor graphs. Many authors have written on aspects of this problem, and, while it is not our purpose to provide a complete reference list, we encourage the interested reader to look at [AL99], [AMY03], [AM07b], [CSWSS12], [CHSW10], [DD05], [LaG16], [LaG08], [Mul02], [Red07], [Smi06] and/or [Yao08].

The "isomorphism problem," on the other hand, is the problem concerning the degree to which $\Gamma(R) \cong \Gamma(S)$ implies that $R \cong S$. Practically, this involves determining which ring-theoretic properties of $R$ can be determined from $\Gamma(R)$. Again, with no claim to completeness, we will briefly highlight some of the progress that has been made in this direction. 
In [AM04], the authors prove, among other things, that if $R$ is a commutative finite reduced ring and if $S$ is a ring which is not a domain, then, with a few minor exceptions, $R \cong S$ if and only if $\Gamma(R) \cong \Gamma(S)$. Results of this type for noncommutative rings are given in [AM06] and [AM07a]. In [SW11], the authors study a variant (originally defined in [Mul02]) called the "graph of equivalence classes of zero-divisors", in which the vertices are equivalence classes of zero-divisors (with equivalence given by equality of annihilators). This structure is quite useful; in particular, it provides information about the associated primes of the underlying ring.

Recently, in [BR11a] and [BR11b], the authors define and investigate an ideal-theoretic version of the zero-divisor graph, called the annihilating-ideal graph of a commutative ring. If $R$ is a commutative ring, then its annihilating-ideal graph, $\mathbb{A} \mathbb{G}(R)$, is the graph whose vertices are the nonzero ideals of $R$ which have nontrivial annihilators, and in which there is an edge between $I$ and $J$ if $I J=0($ and $I \neq J)$. The annihilating-ideal graph is a natural object of study, since many facts about zero-divisors are easily expressed in the language of ideals. Further, as with the graph of equivalence classes of zero-divisors (see [Mul02] and [SW11]), the annihilating-ideal graph eliminates some of the redundancy of the standard zero-divisor graph, and encodes some the the ring theoretic information more efficiently. On the other hand, since the annihilator-ideal graph reflects only information about the ideals of a ring, it cannot make certain fine distinctions between nonisomorphic rings which have very similar zero-divisor behavior. For example, $\mathbb{A} \mathbb{G}\left(\mathbb{Z}_{16}\right)$ and $\mathbb{A} \mathbb{G}\left(\mathbb{Z}_{81}\right)$ are isomorphic as graphs (each is a path of length 3 ) despite the fact that $\mathbb{Z}_{16}$ and $\mathbb{Z}_{81}$ are not isomorphic as rings. In fact, any artinian local PIR (principal ideal ring) whose maximal ideal has index of nilpotence equal to 4 will have this same annihilating-ideal graph. On the other hand, every commutative ring whose annihilating-ideal graph is a path of length 3 must be an artinian local PIR whose maximal ideal has index of nilpotence equal to 4 .

To approach the isomorphism question, we will therefore need to relax our notion of "sameness" for rings. A more reasonable definition involves isomorphism of ideal lattices (see Definition 2.21). In Section 2, we prove that, in the case of commutative artinian rings, the annihilating-ideal graph can distinguish the class of PIRs from the class of nonPIRs; moreover (with one small exception), if $R$ and $S$ are commutative artinian PIRs, then $\mathbb{A} \mathbb{G}(R) \cong \mathbb{A} \mathbb{G}(S)$ precisely when $R$ and $S$ have isomorphic ideal lattices. We further provide examples which indicate the difficulties one might encounter in trying to generalize such a result to a wider class of rings. In addition, we show that the property of being a dual ring is reflected in the annihilating-ideal graph. In Section 3, we extend the known realization results, characterizing annihilating-ideal graphs with six or fewer vertices.

In this article, all rings are assumed to be commutative and unital. In addition, we will restrict our attention primarily to rings whose annihilating-ideal graphs are finite (though some of our results extend easily to the infinite case). Following [BR11a, Theorem 1.4], we will therefore focus on commutative rings with finitely many ideals (purposely ignoring integral domains that are not fields, since their annihilating-ideal graphs, while finite, are trivial). Since all such rings are artinian, we recall two basic facts about commutative artinian rings. First, any commutative artinian ring is a finite direct product of artinian local rings. Second, every artinian PIR must (being a direct product of artinian local PIRs) have only finitely many ideals. See Proposition 2.5 for further discussion. For basic facts about commutative algebra, we refer the reader to [AM69]. On the graph theory side, we 
will consider only graphs without loops (i.e. an edge must connect two distinct vertices). Our terminology will be restricted to basic notions, and will follow standard practice. In particular, we will use $K_{n}$ to refer to the complete graph on $n$ vertices and $P_{n}$ to refer to a path of $n$ vertices. Finally, we note that we will often have occasion to talk simultaneously about ideals as subsets of a ring and as vertices of a graph. In places where there is a possible risk of confusion, we will let $[I]$ refer to the vertex of $\mathbb{A} \mathbb{G}(R)$ which corresponds to the ideal $I$ of $R$.

\section{The Isomorphism Problem: Extracting Ring Theory From the Graph}

As stated in the Introduction, our goal in this section revolves around extracting algebraic properties of $R$ from the graph theory of $\mathbb{A} G(R)$. We begin with some basic algebra. Although these results are surely well-known (in some cases, in greater generality), we include them here for the sake of completeness.

This first result can be found in [Hun68, Proposition 4].

Lemma 2.1. Suppose that $R$ is a commutative local artinian ring whose maximal ideal, $M$, is principal. Then $R$ is a PIR.

Proof. We know that $M=(a)$ for some element $a \in R$. Let $b$ be any nonzero element of $R$. Since $M$ is nilpotent, there is a smallest nonnegative integer $k$ such that $b \in M^{k}$ and $b \notin M^{k+1}$. We claim that $(b)=M^{k}$. It is clear that $(b) \subseteq M^{k}$. Therefore, $b=u a^{k}$ for some element $u \in R$. If $u$ were not a unit, then $u \in M$, which means that $b \in M^{k+1}$, a contradiction. Therefore, $u$ is a unit, which implies that $(b)=M^{k}$. Suppose now that $I$ is any nonzero ideal of $R$. We know that $I$ is finitely generated; write $I=\left(b_{1}, \ldots, b_{n}\right)$. Since, for each $i$, there is a $k_{i}$ such that $\left(b_{i}\right)=M^{k_{i}}$, we see that $I=M^{\min \left(k_{i}\right)}$.

This next lemma is a straightforward verification.

Lemma 2.2. Suppose that $R$ is a commutative local ring with maximal ideal $M$, let $k$ be a nonnegative integer, and let $I$ be an additive subgroup of $R$ such that $M^{k+1} \subseteq I \subseteq M^{k}$. Then $I$ is an ideal of $R$ if and only if $I / M^{k+1}$ is an $R / M$-subspace of $M^{k} / M^{k+1}$

Our final algebraic preliminary is a straightforward application of Nakayama's Lemma.

Lemma 2.3. Suppose that $R$ is a commutative local ring (which is not a field), and suppose that the maximal ideal, $M$, is finitely generated. Then $M$ is principal if and only if $M / M^{2}$ is a one-dimensional vector space over $R / M$.

Proof. Apply Nakayama's Lemma.

We are now ready to commence work on the "Isomorphism Problem". We begin with a general result about maximal ideals. We note here that the annihilating-ideal graph of a commutative ring is always a connected graph by [BR11a, Theorem 2.1]. In what follows, this fact will be used freely and without further mention. 
Lemma 2.4. Let $R$ be a commutative ring with the property that every proper ideal has a nonzero annihilator (in particular, this is the case if $R$ is artinian), and suppose further that $\mathbb{A} \mathbb{G}(R) \neq K_{2}$. Suppose that $\mathbb{A} \mathbb{G}(R)$ has a vertex $[I]$ with the property that, of the vertices adjacent to $[I]$, exactly one vertex, called $[M]$, has degree 1 . Then $M$ is a maximal ideal, $I$ is a minimal ideal, and $I=\operatorname{ann}(M)$.

Proof. Suppose instead that there is a proper ideal $N$ of $R$ such that $M \subset N$. Let $J=$ $\operatorname{ann}(N) \neq 0$. Therefore, $J M=0$. Since $[M]$ has degree 1, it must either be the case that $J=I$ or $J=M$. Suppose first that $J=I$. Since $[N]$ is, therefore, adjacent to $[I]$ and distinct from $[M]$, we know that $[N]$ has degree at least 2 . There must therefore be an ideal $A$, different from $I$, such that $[N]$ is adjacent to $[A]$. But then $N A=0$, which implies that $M A=0$. Since $A$ equals neither $I$ nor $M$, this is a contradiction.

Suppose, on the other hand, that $J=M$. Then $M N=0$, which means that $I=N$. Since $\mathbb{A G}(R) \neq K_{2}$, there is some other ideal $B$, distinct from both $M$ and $N$, such that $[B]$ is adjacent to $[N]$. Therefore, $N B=0$, which means that $M B=0$, a contradiction. Thus $M$ must be maximal.

We now show that $I$ is minimal. Suppose that there is some nonzero ideal $K$ such that $K \subset I$. Since $M I=0$, we must have $M K=0$. This is only possible if $K=M$. Since $\mathbb{A} \mathbb{G}(R) \neq K_{2}$, then there is some ideal $C$, distinct from both $I$ and $M$, such that $[I]$ is adjacent to $[C]$. Since $C I=0$, we have $C K=0$, which is a contradiction since $[K]$, being equal to $[M]$, has degree 1 . Therefore, $I$ is minimal.

Finally, we prove that $I=\operatorname{ann}(M)$. If this is not the case, then we must have $\operatorname{ann}(M)=$ $M$, since $[M]$ is adjacent to no vertex other than $[I]$. Since we have assumed that $\mathbb{A} \mathbb{G}(R) \neq$ $K_{2}$, we may assume that there is an ideal $D$, distinct from both $M$ and $I$ such that $[D]$ is adjacent to $[I]$. Since $D I=0, D \subseteq \operatorname{ann}(I)=M$. As we have assumed that $M=\operatorname{ann}(M)$, this implies that $D \subseteq \operatorname{ann}(M)$ and, thus, that $[D]$ is adjacent to $[M]$, a contradiction. We conclude that $I=\operatorname{ann}(M)$.

As we indicated in the introduction, our primary focus will be on finite annihilating-ideal graphs. By [BR11a, Theorem 1.4], a ring has a finite annihilating-ideal graph if and only if it is either an artinian ring (with finitely many ideals) or a domain (which we ignore, except when the ring in question is actually a field). It is well-known that a commutative artinian ring must be isomorphic to a finite direct product of artinian local rings. If $R$ has only finitely many ideals we can say even more.

Proposition 2.5. Suppose that $R$ is a commutative artinian ring such that $R$ has only finitely many ideals. Then $R \cong R_{1} \times R_{2} \times \cdots \times R_{n}$ where each $R_{i}$ is either a commutative local artinian PIR or a finite commutative local ring.

Proof. It suffices to show that if $R$ is a commutative local artinian ring with finitely many ideals, then $R$ is either a PIR or a finite ring. Suppose, then, that $R$ is not a PIR, and let $M$ be the maximal ideal of $R$. By Lemma 2.1 and Lemma 2.3, $M / M^{2}$ has dimension at least 2 over $R / M$. If $R / M$ were not finite, then $M / M^{2}$ would have infinitely many distinct subspaces, and (appealing to Lemma 2.2) $R$ would have infinitely many ideals, in contradiction with our hypotheses. Thus $R / M$ must be finite. In fact, for each $k$ (up to the index of nilpotence of $M), M^{k-1} / M^{k}$ must be finite dimensional over $R / M$. Thus $M^{k-1} / M^{k}$ is finite for each $k$, which means that $R$ is finite. 
We next recall what is already known about rings with small annihilating-ideal graphs.

Remark 2.6. The non-empty annihilating ideal graphs with four or fewer vertices have been completely classified in [BR11a, Corollary 2.9] (graphs with three of fewer vertices) and $\left[\mathrm{AAB}^{+} 14\right.$, Theorem 12] (graphs with four vertices). As we develop our results, the ability to exclude small cases will be invaluable to us; to that end, we paraphrase the known classification results here.

1. $\mathbb{A} \mathbb{G}(R)$ is a graph with one vertex if and only if $R$ is a local artinian non-field PIR whose maximal ideal is square nilpotent.

2. $\mathbb{A} \mathbb{G}(R)$ is a graph with two vertices if and only if $R$ is either a product of two fields or $R$ is a local artinian PIR whose maximal ideal has index of nilpotence equal to 3 . In each of these cases, $\mathbb{A} \mathbb{G}(R) \cong K_{2}$.

3. $\mathbb{A} \mathbb{G}(R)$ is a graph with three vertices if and only if $R$ is a local artinian PIR whose maximal ideal has index of nilpotence equal to 4 . In this case, $\mathbb{A} \mathbb{G}(R) \cong P_{3}$.

4. $\mathbb{A} \mathbb{G}(R)$ is a graph with four vertices if and only if one of the following three conditions holds.

(a) $R$ is a product of a field and a local artinian non-field PIR whose maximal ideal is square nilpotent. In this case, $\mathbb{A} \mathbb{G}(R) \cong P_{4}$.

(b) $R$ is a local artinian PIR whose maximal ideal has index of nilpotence equal to 5 . In this case, $\mathbb{A} \mathbb{G}(R) \cong\left(K_{2}+K_{1}\right) \vee K_{1}$ (where, for graphs $G$ and $H, G+H$ is the disjoint union of $G$ and $H$, and $G \vee H$ is the graph obtained by starting with the disjoint union of $G$ and $H$ and adding an additional edge connecting every vertex of $G$ to every vertex of $H$ ).

(c) $R$ is a local artinian ring with four nontrivial proper ideals whose maximal ideal squares to zero. In this case, $\mathbb{A} \mathbb{G}(R) \cong K_{4}$.

We draw the reader's attention specifically to the case of a graph with two vertices. Note that $K_{2}$ is the annihilating-ideal graph for two rather different classes of rings. It will turn out that $K_{2}$ is the one and only exception to many of our results, and so we highlight it here.

We begin now by demonstrating how, among artinian rings, the annihlating-ideal graph can be used to distinguish local PIRs from other rings. By Remark 2.6, we note that this is already possible (with the exception of the two-vertex case) for graphs with fewer than four vertices. The restriction below to graphs with at least four vertices is, therefore, not problematic.

Theorem 2.7. Let $R$ be an artinian commutative ring such that $\mathbb{A} \mathbb{G}(R)$ has $n$ vertices $(n \geq 4)$. Then $R$ is a local PIR if and only if $\mathbb{A} \mathbb{G}(R)$ has exactly one vertex of degree 1 , at least one vertex of degree $n-1$ and at least one vertex of degree $n-2$. In this case, the index of nilpotence of the maximal ideal of $R$ is $n+1$.

Proof. Suppose first that $R$ is a local artinian PIR with maximal ideal $M$. Since $\mathbb{A} \mathbb{G}(R)$ has $n$ vertices, it must be the case that the index of nilpotence of $M$ is $n+1$ and that the 
vertices of $\mathbb{A} \mathbb{G}(R)$ are $[M],\left[M^{2}\right], \ldots,\left[M^{n}\right]$. Observe, then, that $[M]$ is the only vertex of degree 1 , that $\left[M^{n}\right]$ has degree $n-1$ and that $\left[M^{n-1}\right]$ has degree $n-2$.

Suppose, on the other hand, that $[M]$ is the vertex of degree 1 , that $[N]$ is a vertex of degree $n-1$ and that $[K]$ is a vertex of degree $n-2$. By [BR11a, Corollary 2.4], we know that $R$ is local. By Lemma 2.4, $M$ is the maximal ideal of $R$, and $N$ is minimal. By Lemma 2.1, it suffices to show that $M$ is a principal ideal. Suppose instead that $M$ is not principal. By Lemma 2.3, the $R / M$-dimension of $M / M^{2}$ is at least 2. This means that $M / M^{2}$ must have at least 3 nontrivial proper subspaces. By Lemma 2.2, there must, therefore, be at least three distinct ideals, say $A, B$ and $C$, each maximal with respect to being properly contained in $M$. Since $[K]$ has degree $n-2$ and $[K]$ is not adjacent to $[M]$, it must be the case that $[K]$ is adjacent to at least two of $[A],[B]$ and $[C]$ (it being possible that $[K]$ is equal to one of them). Suppose, without loss of generality, that $K A=0=K B$. Since $A+B=M$, we have $K M=0$, contradicting the fact that $[K]$ is not adjacent to $[M]$. Thus $R$ is a PIR.

Note that Theorem 2.7 shows that, among commutative artinian rings, the annihilatingideal graph distinguishes between those which are local PIRs and those which are not (with, as usual, the exception of the $K_{2}$ case). We now turn our attention to extending this result to a wider class of rings. In order to do so, we must first introduce some terminology (specialized somewhat to the commutative case).

A commutative ring $R$ is called dual if $\operatorname{ann}(\operatorname{ann}(A))=A$ for every ideal $A$ if $R$. An artinian dual ring is called a Quasi-Frobenius (QF) ring. A commutative ring is called mininjective if $\operatorname{ann}(\operatorname{ann}(N))=N$ for every minimal ideal $N$ (see [NY97, Lemma 1.1]).

Lemma 2.8. Let $R$ be an artinian commutative ring. The following are equivalent:

1. $R$ is $Q F$.

2. $R$ is dual.

3. $R$ is mininjective.

Proof. The implications $1 \Rightarrow 2$ and $2 \Rightarrow 3$ are immediate. For $3 \Rightarrow 1$, see [Har82, Theorem 13] or [NY97, Corollary 4.8].

The QF rings are important in classical ring theory, and we will see that commutative QF rings have annihilating-ideal graphs with particularly nice properties. In order to describe these properties effectively, we make the following graph-theoretic definitions. We recall that a leaf is a degree 1 vertex.

Definition 2.9. Let $G$ be a graph, and let $v$ be a vertex of $G$. If $v$ is a leaf, we will call the unique vertex to which it is adjacent its stem. We will say that a graph $G$ has property $(*)$ if it satisfies each of the following three conditions:

1. $G$ has at least one leaf.

2. No two leaves of $G$ have the same stem.

3. Every nonstem vertex of $G$ is adjacent to at least one stem. 

$(*)$.

Our next result shows what we can say about a ring $R$ for which $\mathbb{A} \mathbb{G}(R)$ has property

Theorem 2.10. Suppose that $R$ is an artinian commutative ring, suppose that $\mathbb{A} \mathbb{G}(R)$ has property $(*)$ and suppose that $\mathbb{A} \mathbb{G}(R) \neq K_{2}$. Then $[M]$ is a leaf of $\mathbb{A} \mathbb{G}(R)$ if and only if $M$ is a maximal ideal of $R$. In particular, if $\mathbb{A} \mathbb{G}(R)$ has $n$ leaves then $R$ has exactly $n$ maximal ideals.

Proof. By Lemma 2.4, each leaf corresponds to a maximal ideal of $R$. It remains to be shown that ideals of $R$ that do not correspond to leaves cannot be maximal. Let $I$ be an ideal of $R$ such that $[I]$ is not a leaf. If $[I]$ is a stem, then by Lemma 2.4, $I$ is minimal. Note that a commutative artinian ring which contains an ideal which is simultaneously maximal and minimal must be either a direct product of two fields or a local nonfield PIR whose maximal ideal is square nilpotent. Since neither of these rings satisfies our hypotheses (the annihilating-ideal graph of the former is isomorphic to $K_{2}$; that of the latter consists of a single vertex), we see that $I$ cannot be maximal. On the other hand, if $[I]$ is not a stem, then $[I]$ must be adjacent to some stem $[N]$ since $\mathbb{A} \mathbb{G}(R)$ has property $(*)$. Suppose that $[M]$ is the leaf which corresponds to the stem $[N]$; as above, we know that $M$ is maximal and, therefore, that $M=\operatorname{ann}(N)$. Since $I N=0, I$ must be contained in $M$, which means that $I$ cannot be maximal.

Theorem 2.11. Let $R$ be an artinian commutative ring such that $\mathbb{A} \mathbb{G}(R)$ has property $(*)$. Then $R$ is $Q F$.

Proof. It is easy to check that $R$ is QF if $\mathbb{A} \mathbb{G}(R) \cong K_{2}$ (see Remark 2.6), so we may assume for the rest of the proof that $\mathbb{A} \mathbb{G}(R) \neq K_{2}$. By Lemma 2.8, it suffices to show that $R$ is mininjective. We first claim that every minimal ideal of $R$ must correspond to a stem in $\mathbb{A} \mathbb{G}(R)$. Indeed, suppose instead that $I$ is a minimal ideal of $R$, but that $[I]$ is not a stem. Since $\operatorname{ann}(I)$ is maximal, $[\operatorname{ann}(I)]$ must be a leaf, by Theorem 2.10. Since $[I]$ is not a stem, and since $I \cdot \operatorname{ann}(I)=0$, it must be the case that $I=\operatorname{ann}(I)$. Thus, $I$ is both maximal and minimal. Suppose that $[N]$ is the stem corresponding to $[\operatorname{ann}(I)]$. Since $\operatorname{ann}(I)=I, N I=0$, which means that $N \subseteq \operatorname{ann}(I)=I$. Since $I$ is minimal, $N=I$, which is a contradiction. Therefore, the minimal ideals of $R$ correspond precisely to the stems of $\mathbb{A} \mathbb{G}(R)$.

Suppose now that $B$ is a minimal ideal of $R$. By what we have just shown, $[B]$ is a stem. Let $[M]$ be its leaf. By Lemma 2.4, $M$ is maximal (implying that $\operatorname{ann}(B)=M$ ) and $\operatorname{ann}(M)=B$. Therefore, $\operatorname{ann}(\operatorname{ann}(B))=\operatorname{ann}(M)=B$, which shows that $R$ is mininjective and, therefore, that $R$ is $\mathrm{QF}$.

We will now show that, in the nonlocal case, the converse to Theorem 2.11 also holds. In the local case, as we will show in Proposition 2.16, we will still be able to characterize the $\mathrm{QF}$ rings, but our characterization will have a slightly different form.

Lemma 2.12. Let $R$ be a commutative $Q F$ ring which is not local. Then a vertex $[M]$ in $\mathbb{A} \mathbb{G}(R)$ is a leaf if and only if $M$ is a maximal ideal in $R$.

Proof. Write $R=R_{1} \times \cdots \times R_{n}$ for suitable local rings $R_{i}(n \geq 2)$. For each $i$, let $M_{i}$ be the maximal ideal of $R_{i}$, and let $\mathcal{M}_{i}$ be the ideal $\left(R_{1}, \ldots, M_{i}, \ldots, R_{n}\right)$ of $R$. We will first prove 
that $\left[\mathcal{M}_{i}\right]$ is a leaf for each $i$. Since $R$ is $\mathrm{QF}, \operatorname{ann}\left(\mathcal{M}_{i}\right)=\left(0, \ldots, \operatorname{ann}\left(M_{i}\right), \ldots, 0\right)$ is a minimal ideal. Note further that $\operatorname{ann}\left(\mathcal{M}_{i}\right) \neq \mathcal{M}_{i}$. Therefore, $\left[\mathcal{M}_{i}\right]$ is adjacent only to $\left[\operatorname{ann}\left(\mathcal{M}_{i}\right)\right]$.

Suppose now that $I$ is an ideal of $R$ such that $[I]$ is a leaf. Write $I=\left(A_{1}, \ldots, A_{n}\right)$ for suitable ideals $A_{i}$ if $R_{i}$. If $I$ is not maximal, then either $A_{i} \neq R_{i}$ for at least two indices, or $A_{i} \subset M_{i}$ for at least one index. In the first case, we may assume without loss of generality that $A_{1} \neq R_{1}$ and $A_{2} \neq R_{2}$. Then $I$ is annihilated by $\left(\operatorname{ann}\left(A_{1}\right), 0, \ldots, 0\right)$, $\left(0, \operatorname{ann}\left(A_{2}\right), \ldots, 0\right)$ and $\left(\operatorname{ann}\left(A_{1}\right), \operatorname{ann}\left(A_{2}\right), \ldots, 0\right)$, all three of which must be distinct and nonzero. Even though $I$, itself, might be equal to one of these three ideals, we can still conclude that $[I]$ has degree at least 2 . In the second case, on the other hand, we know that $I$ is annihilated by $\left(\operatorname{ann}\left(M_{1}\right), \ldots, 0\right)$ and $\left(\operatorname{ann}\left(A_{1}\right), \ldots, 0\right)$, which are different (and nonzero) since $R$ is QF. If $I$ is distinct from these two ideals, then we have established in this case that the degree of $[I]$ is at least 2 . If $I$ does happen to be equal to one of these two ideals, then we simply note that $I$ must also be annihilated by $\left(\operatorname{ann}\left(M_{1}\right), R_{2}, \ldots, 0\right)$. Thus $[I]$ must have degree at least 2. Therefore, if $[I]$ is a leaf, then $I$ must be maximal.

Remark 2.13. Note that neither the "nonlocal" nor "dual" hypothesis may be removed from Lemma 2.12. To show the necessity of the nonlocality, let $R$ be a local PIR whose maximal ideal has index of nilpotence 3 (e.g. $R=\mathbb{Z}_{8}$ ). In this case, $\mathbb{A} \mathbb{G}(R) \cong K_{2}$, which has two leaves. However, since we have seen that $K_{2}$ is often a special case, we will give another example. Let $S=\mathbb{Z}_{2}[x, y] /\left(x^{2}, y^{2}\right)$. Then $S$ is local and QF, but $\mathbb{A} \mathbb{G}(S)$ has 4 leaves (see Figure 1, which follows Example 2.23).

To show the necessity of the "dual" hypothesis, let $T=\mathbb{Z}_{2}[x, y] /(x, y)^{2} \times \mathbb{Z}_{2}$. Then $T$ is not $\mathrm{QF}(\operatorname{since} \operatorname{ann}(\operatorname{ann}((x), 0))=((x, y), 0))$. Then $S$ has two maximal ideals, but $\mathbb{A} \mathbb{G}(S)$ has only one leaf (corresponding to the ideal $\mathbb{Z}_{2}[x, y] /(x, y)^{2} \times\{0\}$ ).

Lemma 2.14. If $R$ is a commutative QF ring which is not local, then no two leaves of $\mathbb{A} \mathbb{G}(R)$ can have the same stem.

Proof. Suppose instead that $M$ and $N$ are distinct ideals of $R$ such that $[M]$ and $[N]$ are leaves which share a stem, which we will call $[K]$. By Lemma 2.12, $M$ and $N$ are both maximal. As was observed in the proof of Lemma 2.12, $\operatorname{ann}(M)=K=\operatorname{ann}(N)$. Since $R$ is $\mathrm{QF}, M=N$, which is a contradiction.

We can now establish the converse to Theorem 2.11 in the nonlocal case.

Theorem 2.15. Let $R$ be a commutative $Q F$ ring which is not local. Then $\mathbb{A} \mathbb{G}(R)$ has property $(*)$.

Proof. By Lemma 2.12, $\mathbb{A} \mathbb{G}(R)$ has at least one leaf. Further, by Lemma 2.14, no two leaves of $\mathbb{A} \mathbb{G}(R)$ have the same stem. Finally, suppose that $I$ is any ideal of $R$. Then $I \subseteq M$ for some maximal ideal $M$ of $R$. By Lemma $2.12,[M]$ is a leaf. Thus $[I]$ is adjacent to $[\operatorname{ann}(M)]$, which is a stem.

In the local case, we have the following, slightly different, characterization.

Proposition 2.16. Let $R$ be a commutative artinian local ring with maximal ideal $M$. Then $R$ is $Q F$ if and only if either $\mathbb{A} \mathbb{G}(R)$ is empty, $\mathbb{A} \mathbb{G}(R)$ is a singleton, or $\mathbb{A} \mathbb{G}(R)$ has at least one leaf. 
Proof. We first note that $R$ is a field if and only if $\mathbb{A} \mathbb{G}(R)$ is empty and that $R$ is a nonfield PIR with $M^{2}=0$ if and only if $\mathbb{A} \mathbb{G}(R)$ is a singleton. We assume now that $\mathbb{A} \mathbb{G}(R)$ has at least 2 vertices.

Suppose first that $R$ is $\mathrm{QF}$. Since $\mathbb{A} \mathbb{G}(R)$ has at least 2 vertices, we know that $M \neq$ $\operatorname{ann}(M)$. Since $\operatorname{ann}(M)$ is minimal, $[M]$ is a leaf.

On the other hand, suppose that $\mathbb{A} \mathbb{G}(R)$ has at least one leaf, $\left[M^{\prime}\right]$. By Lemma 2.8, it suffices to show that $R$ is mininjective. Let $[N]$ be the stem of $\left[M^{\prime}\right]$. By [BR11a, Corollary $2.4]$, we know that $[N]$ must be adjacent to every other vertex of $\mathbb{A} \mathbb{G}(R)$. In particular, $[M]$ is adjacent to $[N]$. We claim that $[M]$ must be a leaf. If not, then the degree of $[M]$ is at least 2 , which means that $[M]$ is adjacent to some vertex $[A]$, distinct from $[N]$. But then $M A=0$, which implies that $M^{\prime} A=0$, a contadiction. Therefore, $[M]$ is a leaf. Since $M \neq \operatorname{ann}(M)$, we must have $\operatorname{ann}(M)=N$. Since $N$ is then the only possible minimal ideal (any minimal ideal of $R$ is annihilated by $M$ ), and since $\operatorname{ann}(N)=M$, we know that $R$ is mininjective, which proves that $R$ is $\mathrm{QF}$.

Corollary 2.17. Let $R$ be a commutative artinian ring. Then $R$ is $Q F$ if and only if one of the following is true:

1. $\mathbb{A} \mathbb{G}(R)$ is empty.

2. $\mathbb{A} \mathbb{G}(R)$ consists of a single vertex.

3. $\mathbb{A} \mathbb{G}(R)$ contains a vertex which is adjacent to every other vertex and $\mathbb{A} \mathbb{G}(R)$ contains at least one leaf.

4. $\mathbb{A} \mathbb{G}(R)$ does not contain a vertex which is adjacent to every other vertex and $\mathbb{A} \mathbb{G}(R)$ satisfies property $(*)$.

Proof. Note first that (reasoning as in the proof of Propositon 2.16), in the commutative artinian case, $\mathbb{A} \mathbb{G}(R)$ is empty precisely when $R$ is a field (or the zero ring), and $\mathbb{A} \mathbb{G}(R)$ has a single vertex if and only if $R$ has a single nonzero proper ideal. In both of these cases, $R$ is QF. Suppose that $\mathbb{A} \mathbb{G}(R)$ contains a vertex which is adjacent to every other vertex. By [BR11a, Corollary 2.4], $R$ is either a product of two fields (and, therefore, QF) or $R$ is local. By Proposition 2.16, $R$ is QF. Finally, if $\mathbb{A} \mathbb{G}(R)$ satisfies property (*), then $R$ is QF by Theorem 2.11 .

Suppose, on the other hand, that $R$ is QF. Proposition 2.16 and [BR11a, Corollary 2.4] (in the local case) and Theorem 2.15 (in the nonlocal case) establish the converse.

To summarize our results so far, we have shown that, in certain cases, the annihilatingideal graph can detect a variety of ring-theoretic information (e.g. maximal and minimal ideals, whether a ring is local, whether a ring is QF, etc.). Our second main result of this section will show that, among the commutative QF rings, the PIRs can be distinguished from the non-PIRs. In addition, we will show that, with the usual exception (rings whose annihilating-ideal graphs are isomorphic to $K_{2}$ ), the annihilating-ideal graph can distinguish between PIRs with different ideal lattices. We begin with a preparatory lemma. 
Lemma 2.18. Let $R$ be a commutative $Q F$ ring which is not local. Suppose further that $\mathbb{A} \mathbb{G}(R) \nRightarrow P_{4}$. Write $R=S \times T$ for some nonzero ring $S$ and some nonzero local ring $T$. Let $I$ be the minimal ideal of $T$ (i.e. the annihilator of the maximal ideal of $T$ ). Then $T$ is a field if and only if $[(0, I)]$ has no neighbor whose degree is 2.

Proof. Suppose first that $T$ is a field. Then $I=T$. We claim that $[(0, T)]$ is not adjacent to any vertex whose degree is 2 . To begin, we know that $[(0, T)]$ is adjacent to $[(S, 0)]$, whose degree is 1 by Lemma 2.12. Every other neighbor of $[(0, T)]$ has the form $[(A, 0)]$ for some proper ideal $A$ of $S$. Note that $(\operatorname{ann}(A), T),(0, T)$ are distinct ideals, different from $(A, 0)$, that annihilate $(A, 0)$. We now consider two cases. If $A$ is maximal in $S$ and $\operatorname{ann}(A) \neq A$, then $(\operatorname{ann}(A), 0)$ is a third distinct ideal that annihilates $(A, 0)$. In this case, the degree of $[(A, 0)]$ is at least 3. If, on the other hand, $A$ is maximal and $\operatorname{ann}(A)=A$, then $A$ is the only nontrivial proper ideal of $S$, in which case $\mathbb{A G}(R) \cong P_{4}$. If $A$ is not maximal, then let $M$ be a maximal ideal of $S$ which contains $A$. Then $(\operatorname{ann}(M), T)$ is a third distinct ideal which annihilates $(A, 0)$, which proves again that the degree of $[(A, 0)]$ is at least 3 .

Suppose now that $T$ is not a field. Let $N$ be the maximal ideal of $T$, and let $J \subseteq N$ be an ideal of $T$, maximal with respect to being unequal to $N$. Note that $[(S, J)]$ is adjacent to $[(0, I)]$. We claim that $[(S, J)]$ has degree 2 . It is clear that $(S, J)$ is annihilated by $(0, I)$ and by $(0, \operatorname{ann}(J))$, and that these are distinct. Suppose that $(A, B)$ is another nonzero ideal which annihilates $(S, J)$. Then $A=0$ and $B \subseteq \operatorname{ann}(J)$. Since $R$ is QF, the latter implies that $J \subseteq \operatorname{ann}(B)$, which means that either $\operatorname{ann}(B)=J$ or $\operatorname{ann}(B)=N$. But then $B=\operatorname{ann}(J)$ or $B=\operatorname{ann}(N)$. Therefore, $[(S, J)]$ has degree 2 .

We can now prove our main theorem, inspired in part by [AM04, Theorem 4]. Although the statement of the theorem seems somewhat technical, it is based on a relatively straightforward idea. If $R$ is a commutative artinian ring, then $R$ can be expressed as a finite direct product of commutative local artinian rings. If $\mathbb{A} \mathbb{G}(R)$ satisfies the condition $(*)$, then our theorem provides an algorithm for extracting the annihilating-ideal graph of each of these local factors from $\mathbb{A} \mathbb{G}(R)$. The special cases merely reflect the fact that our algorithm needs a slight modification in the case where the ring $R$ is the direct product of a field with another local ring.

Note that, since annihilating-ideal graphs with four vertices or fewer have been completely classified (see Remark 2.6), our restriction to graphs with at least five vertices poses no problem.

Theorem 2.19. Let $R$ be an artinian commutative ring. Suppose that $\mathbb{A} \mathbb{G}(R)$ has at least 5 vertices, suppose that $\mathbb{A} \mathbb{G}(R)$ has $n$ leaves $(2 \leq n<\infty)$, and suppose that $\mathbb{A} \mathbb{G}(R)$ satisfies $(*)$. Let $\left[\mathcal{M}_{1}\right], \ldots,\left[\mathcal{M}_{n}\right]$ denote the leaves. For each $i$, let $\left[\mathcal{N}_{i}\right]$ be the stem of $\left[\mathcal{M}_{i}\right]$. For each $i$, let

$$
S_{i}=\left\{[A] \mid[A] \neq\left[\mathcal{N}_{j}\right] \forall j \text {, and }[A] \text { is adjacent to }\left[\mathcal{N}_{i}\right] \text { but not to }\left[\mathcal{N}_{j}\right] \forall j \neq i\right\}
$$

and

$$
T_{i}=\left\{[B] \mid[B] \text { is adjacent to at least one vertex in } S_{i}\right\}
$$

Let $H_{i}$ be the subgraph of $\mathbb{A} \mathbb{G}(R)$ induced by the vertices in $T_{i}$. 
Then $H_{i}$ will either be a connected graph (possibly empty), or it will consist of two connected components, one of which consists of a single vertex.

Define $\hat{H}_{i}$ as follows.

Case 1: If $n \geq 3$ or if $n=2$ and each of $\left[\mathcal{N}_{1}\right]$ and $\left[\mathcal{N}_{2}\right]$ has at least one degree 2 neighbor, then

(a) If $H_{i}$ consists of a single vertex, define $\hat{H}_{i}$ to be the empty graph.

(b) If $H_{i}$ consists of two nonadjacent vertices, define $\hat{H}_{i}$ to be a single vertex.

(c) If $H_{i}$ consists of the union of a single vertex and a larger connected component, define $\hat{H}_{i}$ to be the larger connected component.

Case 2: If $n=2$ and either $\left[\mathcal{N}_{1}\right]$ or $\left[\mathcal{N}_{2}\right]$ has no degree 2 neighbor, then

(a) If $\left[\mathcal{N}_{1}\right]$ has no degree 2 neighbor and $\left[\mathcal{N}_{2}\right]$ does have a degree 2 neighbor, then define $\hat{H}_{1}$ to be the empty graph, and define $\hat{H}_{2}$ to be $H_{2}$.

(b) If $\left[\mathcal{N}_{1}\right]$ does have a degree 2 neighbor and $\left[\mathcal{N}_{2}\right]$ has no degree 2 neighbor, then define $\hat{H}_{1}$ to be $H_{1}$, and define $\hat{H}_{2}$ to be the empty graph.

Then there are local rings $R_{1}, \ldots, R_{n}$ such that $R \cong R_{1} \times \cdots \times R_{n}$ and such that $\hat{H}_{i} \cong \mathbb{A} \mathbb{G}\left(R_{i}\right)$ for each $i$.

Proof. Since $\mathbb{A} \mathbb{G}(R)$ satisfies $(*)$, we may apply Lemma 2.4. Therefore, $\mathcal{M}_{1}, \ldots, \mathcal{M}_{n}$ are maximal, and $\mathcal{N}_{1}, \ldots, \mathcal{N}_{n}$ are minimal. Further, by Theorem 2.10 , the $\mathcal{M}_{i}$ are the only maximal ideals. Following the proof of Theorem 2.11, we can also conclude that the $\mathcal{N}_{i}$ are the only minimal ideals. Since $R$ is an artinian commutative ring with $n$ maximal ideals, we know that $R=R_{1} \times \cdots \times R_{n}$ for suitable local rings $R_{i}$. Let $M_{i}$ denote the maximal ideal of $R_{i}$. Then we may assume (relabeling if necessary) that

$$
\mathcal{M}_{i}=\left(R_{1}, \ldots, M_{i}, \ldots, R_{n}\right)
$$

and

$$
\mathcal{N}_{i}=\left(0, \ldots, \operatorname{ann}\left(M_{i}\right), \ldots, 0\right)
$$

We claim that $S_{i}=\left\{\left[\left(R_{1}, \ldots, A, \ldots, R_{n}\right)\right] \mid A\right.$ is an ideal of $\left.R_{i}\right\}$. On the one hand, $\left(R_{1}, \ldots, A, \ldots, R_{n}\right)$. $\left(0, \ldots, \operatorname{ann}\left(M_{i}\right), \ldots, 0\right)=0$ for every proper ideal $A$ of $R_{i}$. On the other hand, if $I=$ $\left(I_{1}, \ldots, I_{n}\right)$ is an ideal of $R$ such that $I$ does not annihilate $\mathcal{N}_{j}$ for any $j \neq i$, then it must be the case that $I_{j}=R_{j}$ for every $j \neq i$. Note that none of the elements $\left(R_{1}, \ldots, A, \ldots, R_{n}\right)$ can equal one of the $\mathcal{N}_{j}$ for some $j \neq i$ unless $n=2$ and either $R_{1}$ or $R_{2}$ is a field. We assume for now that this is not the case. By Lemma 2.18, this means that we will define $\hat{H}_{i}$ according to Case 1 in the statement of the theorem. Having determined the elements of $S_{i}$, we see immediately that $T_{i}=\left\{[(0, \ldots, B, \ldots, 0)] \mid B\right.$ is an ideal of $\left.R_{i}\right\}$. Suppose now that $[(0, \ldots, B, \ldots, 0)]$ and $[(0, \ldots, C, \ldots, 0)]$ are two elements of $T_{i}$. Note that, as long as neither $B$ nor $C$ is equal to $R_{i}$, then $[(0, \ldots, B, \ldots, 0)]$ is adjacent to $[(0, \ldots, C, \ldots, 0)]$ if and only if $[B]$ is adjacent to $[C]$ in $\mathbb{A} \mathbb{G}\left(R_{i}\right)$. Note also that $\left[\left(0, \ldots, R_{i}, \ldots, 0\right)\right]$ is adjacent to no vertex of $T_{i}$. Therefore, if we define the graph $\hat{H}_{i}$ to be the subgraph of $\mathbb{A} \mathbb{G}\left(R_{i}\right)$ induced by $T_{i}$, with the lone vertex, namely $\left[\left(0, \ldots, R_{i}, \ldots, 0\right)\right]$, removed, then $\hat{H}_{i} \cong \mathbb{A} \mathbb{G}\left(R_{i}\right)$. Note that we have also demonstrated that the sub-cases listed for Case 1 are exhaustive. 
We now deal with the exceptional case that $n=2$ and that either $R_{1}$ or $R_{2}$ is a field. Appealing again to Lemma 2.18, we see that this means that we will define $\hat{H}_{i}$ according to Case 2 above. We assume first that $\left[\mathcal{N}_{1}\right]$ has no degree 2 neighbor but that $\left[\mathcal{N}_{2}\right]$ does. By Lemma 2.18, $R_{1}$ is a field. Then $\mathbb{A} \mathbb{G}\left(R_{1}\right)$ is empty. This means that $S_{2}$ does not contain $\left[\left(R_{1}, 0\right)\right]$ (by definition), which means that $T_{1}$ does not contain $\left[\left(0, R_{2}\right)\right]$. Arguing as we did in Case 1 above, this means that $H_{2}$ will be connected and that $H_{2} \cong \mathbb{A} \mathbb{G}\left(R_{2}\right)$. The proof in the case that $\left[\mathcal{N}_{2}\right]$ has no degree 2 neighbor but $\left[\mathcal{N}_{1}\right]$ does have a degree 2 neighbor is analogous. Note finally that if neither $\left[\mathcal{N}_{1}\right]$ nor $\left[\mathcal{N}_{2}\right]$ has a degree 2 neighbor, then, by Lemma 2.18 , both $R_{1}$ and $R_{2}$ are fields (implying that $\mathbb{A} \mathbb{G}(R) \cong K_{2}$ ), which is impossible given our assumption that $\mathbb{A} \mathbb{G}(R)$ has at least five vertices. Thus the sub-cases of Case 2 are also exhaustive.

Remark 2.20. Theorem 2.19 shows how one can, in certain cases, take the annihilatingideal graph of a direct product of ring and extract from it the annihilating-ideal graphs of the direct factors. We mention that the opposite problem (i.e. determining the annihilating-ideal graph of a product of rings from the annihilating-ideal graphs of the factor rings) presents a few difficulties, which we outline here. If $R$ and $S$ are rings, then the ideals of $R \times S$ have the form $(I, J)$, where $I$ is an ideal of $R$ and $J$ is an ideal of $S$. In general, this suggests that the set of vertices of $\mathbb{A} \mathbb{G}(R \times S)$ should be the cartesian product of the set of vertices of $\mathbb{A} \mathbb{G}(R)$ with the set of vertices of $\mathbb{A} \mathbb{G}(S)$. The issue is that $\mathbb{A} \mathbb{G}(R \times S)$ has vertices, such as $[(R, 0)]$, that do not come in this way from the sets of vertices of $\mathbb{A} \mathbb{G}(R)$ and $\mathbb{A} \mathbb{G}(S)$ (since $[R]$ is not a vertex of $\mathbb{A} \mathbb{G}(R)$, and [0] is not a vertex of $\mathbb{A} \mathbb{G}(S)$ ). This issue seems relatively easy to fix. Determining the edges of $\mathbb{A} \mathbb{G}(R \times S)$ is somewhat more problematic. For example, if $I_{1}$ and $I_{2}$ are ideals of $R$ such that $I_{1} I_{2}=0$ and if $J$ is an ideal of $S$, then $\left[\left(I_{1}, J\right)\right]$ is adjacent to $\left[\left(I_{2}, J\right)\right]$ if and only if $J^{2}=0$. However, determining whether or not the ideal $J$ is square nilpotent seems to be rather difficult from $\mathbb{A} \mathbb{G}(S)$.

Definition 2.21. Let $R$ and $S$ be commutative rings. We will say that $R$ and $S$ are "lattice isomorphic" if their lattices of ideals are isomorphic.

Corollary 2.22. Suppose that $R$ is a commutative artinian PIR whose annihilating-ideal graph is not isomorphic to $K_{2}$ and that $S$ is any commutative ring such that $\mathbb{A} \mathbb{G}(R) \cong \mathbb{A} \mathbb{G}(S)$. Then $S$ is a PIR, and $S$ is lattice-isomorphic to $R$.

Proof. Note first that $R$ has finitely many ideals, which means that $\mathbb{A} \mathbb{G}(R)$ is finite. Thus $\mathbb{A G}(S)$ is finite, and by [BR11a, Theorem 1.4], $S$ is artinian. If $R$ is local, then the result is true by Theorem 2.7, since the ideal lattice of a local artinian PIR is determined by the index of nilpotence of the maximal ideal. We may therefore assume that $R$ is not local. Likewise, if $\mathbb{A} \mathbb{G}(R)$ has four or fewer vertices, then we may appeal to Remark 2.6. We may therefore assume that $R$ is not local and that $\mathbb{A} \mathbb{G}(R)$ has at least 5 vertices. Since an artinian $\mathrm{PIR}$ is $\mathrm{QF}$, we may apply Theorem 2.15 to prove that $\mathbb{A} \mathbb{G}(R)$ has property $(*)$. Therefore, $\mathbb{A} \mathbb{G}(S)$ has property $(*)$. Appealing to Theorem 2.19 , we see that $R \cong R_{1} \times \cdots \times R_{n}$ and that $S \cong S_{1} \times \cdots \times S_{n}$ for suitable local rings $R_{i}$ and $S_{i}$ and that, after suitable relabeling, $\mathbb{A} \mathbb{G}\left(R_{i}\right) \cong \mathbb{A} \mathbb{G}\left(S_{i}\right)$ for each $i$. By Theorem 2.7, we are then done.

It would be desirable to extend Corollary 2.22 to a wider class of rings. The following examples illustrate some of the limitations placed on any possible generalization. 
Example 2.23. The rings $\mathbb{Z}_{2}[x, y] /\left(x^{2}, y^{2}\right)$ (see Figure 1 ) and $\mathbb{Z}_{2}[x, y] /\left(x y, x^{2}-y^{2}\right.$ ) (see Figure 2) have isomorphic ideal lattices, but their annihilating-ideal graphs are not isomorphic. Note that both rings are QF and also local.

On the other hand, the annihilating-ideal graphs of $\mathbb{Z}_{2}[x, y, z] /(x, y, x)^{2}$ and $\mathbb{Z}_{13}[x, y] /(x, y)^{2}$ are isomorphic (both are complete graphs on 15 vertices), but these two rings have very different ideal lattices. Note that neither of these rings is QF.

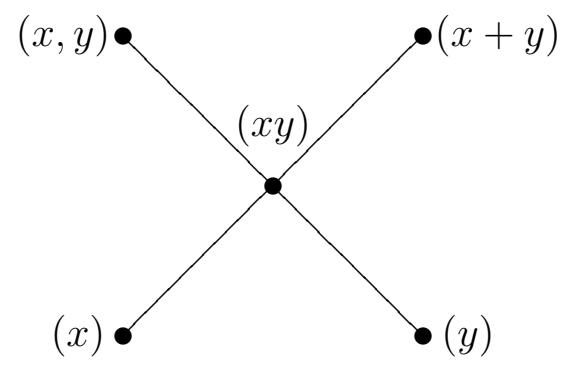

Figure 1: $\mathbb{A} \mathbb{G}\left(\mathbb{Z}_{2}[x, y] /\left(x^{2}, y^{2}\right)\right)$

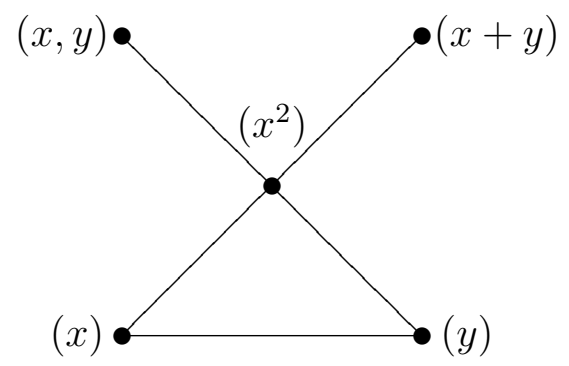

Figure 2: $\mathbb{A} \mathbb{G}\left(\mathbb{Z}_{2}[x, y] /\left(x y, x^{2}-y^{2}\right)\right)$

\section{The Realizability Problem: Extracting Graph The- ory from the Ring}

This section focuses on extending what is known about which (finite) graphs occur as the annihilating-ideal graphs of commutative rings. As we mentioned in Remark 2.6, annihilating-ideal graphs with four or fewer vertices have been completely classified in [BR11a] and $\left[\mathrm{AAB}^{+} 14\right]$. We will extend this work to cover graphs with five or six vertices. We will also determine precisely which finite star graphs can occur as the annihilating-ideal graphs of a commutative ring, building on [BR11a, Theorem 2.6].

We begin with a simple counting statement.

Lemma 3.1. Let $R$ be a commutative ring with finitely many ideals. Write $R=R_{1} \times \cdots \times R_{n}$ for suitable commutative local rings $R_{1}, \ldots, R_{n}$. Then

$$
|\mathbb{A} \mathbb{G}(R)|=\prod_{i=1}^{n}\left(\left|\mathbb{A} \mathbb{G}\left(R_{i}\right)\right|+2\right)-2
$$


Proof. If $R$ has finitely many ideals, then this number of ideals is equal to $|\mathbb{A} \mathbb{G}(R)|+2$.

The following lemma will also prove useful.

Lemma 3.2. Let $R$ be a finite commutative ring, and let $A$ be a principal ideal of $R$. Then $|A| \cdot|\operatorname{ann}(A)|=|R|$.

Proof. Write $A=(a)$ for some $a \in R$. Then $R / \operatorname{ann}(A) \cong A$, as $R$-modules.

We now complete the classification of the five-vertex annihilating-ideal graphs. In what follows, some of the arguments will involve a count of the subspaces of a finite-dimensional vector space over a finite field. To this end, we recall that if $V$ is an $n$-dimensional vector space over the finite field $\mathbb{F}_{q}$, then the number of subspaces of $V$ of dimension $k(1 \leq k \leq n)$ is given by the $q$-binomial coefficient

$$
\left(\begin{array}{l}
n \\
k
\end{array}\right)_{q}=\frac{\left(1-q^{n}\right)\left(1-q^{n-1}\right) \ldots\left(1-q^{n-k+1}\right)}{(1-q)\left(1-q^{2}\right) \ldots\left(1-q^{k}\right)} .
$$

Theorem 3.3. Suppose that $G$ is a five-vertex graph such that $G \cong \mathbb{A} G(R)$ for some commutative ring $R$. Then $G$ is isomorphic to one of the following four graphs: $K_{5}, \mathbb{A} \mathbb{G}\left(\mathbb{Z}_{2}[x] /\left(x^{6}\right)\right)$ (see Figure 3), $\mathbb{A} \mathbb{G}\left(\mathbb{Z}_{2}[x, y] /\left(x^{2}, y^{2}\right)\right.$ ) (see Figure 1) or $\mathbb{A} \mathbb{G}\left(\mathbb{Z}_{2}[x, y] /\left(x y, x^{2}-y^{2}\right)\right.$ ) (see Figure 2).

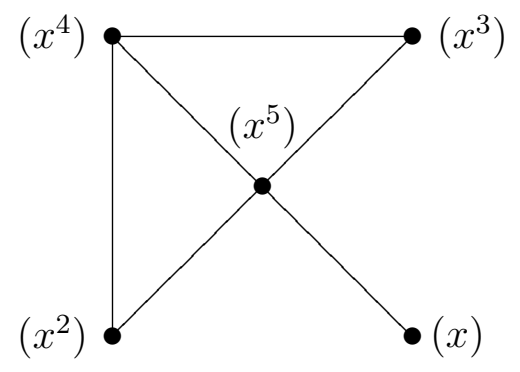

Figure 3: $\mathbb{A} \mathbb{G}\left(\mathbb{Z}_{2}[x] /\left(x^{6}\right)\right)$

Proof. To begin, we know from [BR11a, Theorem 1.4] that $R$ must have precisely seven ideals. Furthermore, Lemma 3.1 shows that $R$ must be local (since 7 is prime). Let $M$ be the maximal ideal of $R$. Consider the $R / M$-vector space $M / M^{2}$. If $M / M^{2}$ has $R / M$ dimension 1, then, by Lemma 2.1 and Lemma $2.3, R$ is a local PIR. In this case, $G$ must be isomorphic to $\mathbb{A} \mathbb{G}\left(\mathbb{Z}_{2}[x] /\left(x^{6}\right)\right)$ by Theorem 2.7 .

Suppose, on the other hand, that the $R / M$-dimension of $M / M^{2}$ is not 1 . By Lemma 2.2, $M / M^{2}$ can have at most $6 R / M$-subspaces. Calculating using the $q$-binomial coefficient, we conclude that one of two things must occur: either $R / M \cong \mathbb{F}_{3}$ and $M^{2}=0$, or $R / M \cong \mathbb{F}_{2}$ and $M^{2} \neq 0$. In the former case, it is immediate that $G \cong K_{5}$.

We now focus on the latter possibility. In this case, there are three ideals strictly between $M$ and $M^{2}$, and every ideal, save $M$, is principal. Moreover, since $\operatorname{ann}\left(M^{2}\right)=M$, Lemma 3.2 shows that $M^{2}$ must have exactly two elements. Let $A, B$ and $C$ be the three ideals which lie 
properly between $M$ and $M^{2}$. Each must have exactly four elements, and the index of each in $R$ must also be 4 . Since each of $A, B$ and $C$ is principal, by Lemma 3.2, the annihilator of each must also have four elements. Therefore, either each of $A, B$ and $C$ is its own annihilator, or two of these ideals form an annihilator pair, while one is its own annihilator. In the former case, the annihilating-ideal graph of $R$ is isomorphic to $\mathbb{A} \mathbb{G}\left(\mathbb{Z}_{2}[x, y] /\left(x^{2}, y^{2}\right)\right)$ (see Figure 1 ); in the latter case, the annihilating-ideal graph of $R$ is isomorphic to $\mathbb{A} \mathbb{G}\left(\mathbb{Z}_{2}[x, y] /\left(x y, x^{2}-y^{2}\right)\right)$ (see Figure 2).

A similar argument can be used to obtain a classification result for six-vertex annihilatingideal graphs.

Theorem 3.4. Suppose that $G$ is a six-vertex graph such that $G \cong \mathbb{A} G(R)$ for some commutative ring $R$. Then $G$ is isomorphic to one of the following six graphs: $\mathbb{A}\left(\mathbb{Z}_{2} \times \mathbb{Z}_{2} \times \mathbb{Z}_{2}\right)$ (see Figure 4), $\mathbb{A} \mathbb{G}\left(\mathbb{Z}_{2}[x] /\left(x^{3}\right) \times \mathbb{Z}_{2}\right)$ (see Figure 5), $K_{6}, \mathbb{A} \mathbb{G}\left(\mathbb{Z}_{2}[x] /\left(x^{7}\right)\right.$ ) (see Figure 6$)$, $\mathbb{A} \mathbb{G}\left(\mathbb{Z}_{3}[x, y] /\left(x y, x^{2}-y^{2}\right)\right)$ (see Figure 7) or $\mathbb{A} \mathbb{G}\left(\mathbb{Z}_{3}[x, y] /\left(x y, x^{2}+y^{2}\right)\right.$ ) (see Figure 8).

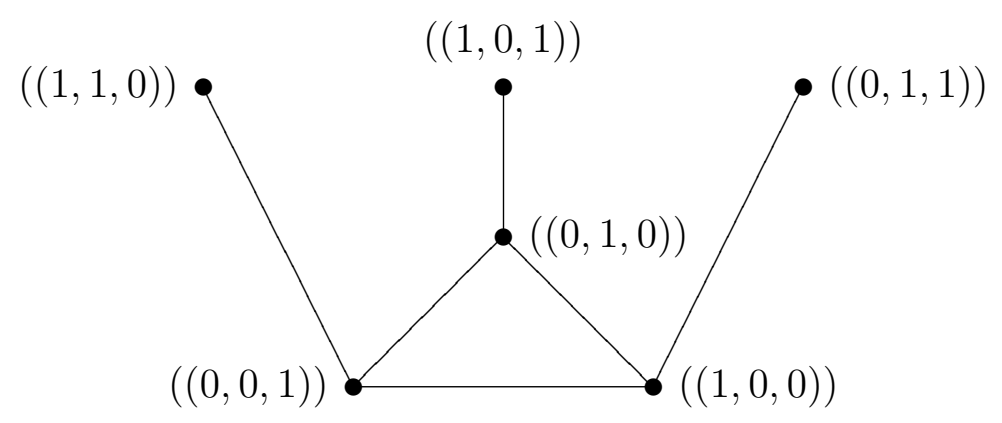

Figure 4: $\mathbb{A} \mathbb{G}\left(\mathbb{Z}_{2} \times \mathbb{Z}_{2} \times \mathbb{Z}_{2}\right)$

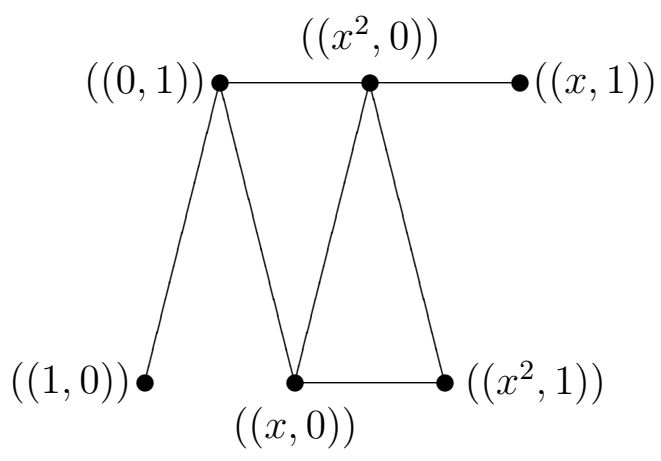

Figure 5: $\mathbb{A} \mathbb{G}\left(\mathbb{Z}_{2}[x] /\left(x^{3}\right) \times \mathbb{Z}_{2}\right)$

Proof. Suppose that $\mathbb{A} \mathbb{G}(R)$ is a graph with six vertices. By [BR11a, Theorem 1.4], we see that $R$ has precisely eight ideals, and by Lemma 3.1, we surmise that $R$ is either local or that $R$ is a nontrivial direct product of local rings. In the latter case, $R$ is either a direct product of two local rings (one of which is a field, and the other of which has four ideals) 


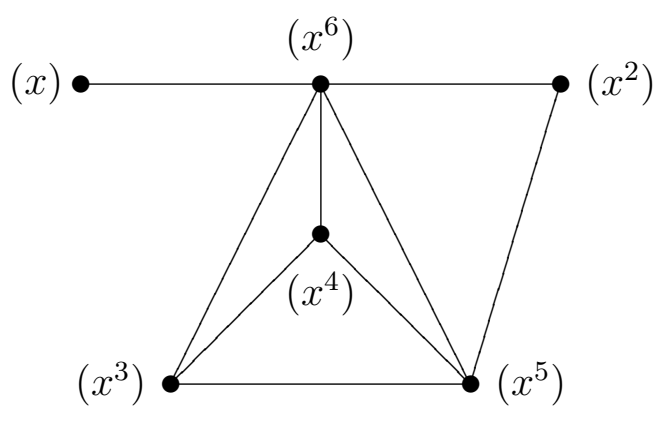

Figure 6: $\mathbb{A} \mathbb{G}\left(\mathbb{Z}_{2}[x] /\left(x^{7}\right)\right)$

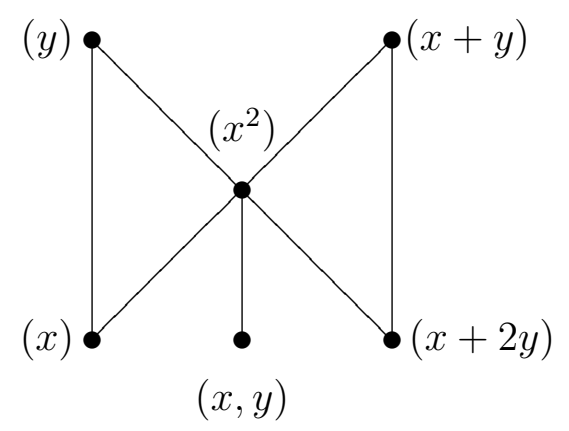

Figure 7: $\mathbb{A} \mathbb{G}\left(\mathbb{Z}_{3}[x, y] /\left(x y, x^{2}-y^{2}\right)\right)$

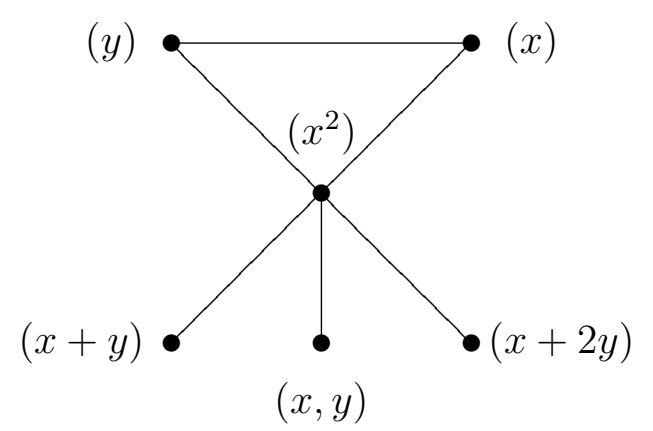

Figure 8: $\mathbb{A} \mathbb{G}\left(\mathbb{Z}_{3}[x, y] /\left(x y, x^{2}+y^{2}\right)\right)$

in which case $\mathbb{A} \mathbb{G}(R)$ is isomorphic to the graph in Figure 5, or $R$ is the direct product of three fields, in which case $\mathbb{A} \mathbb{G}(R)$ is isomorphic to the graph in Figure 4.

We may now assume that $R$ is local with maximal ideal $M$. As we did in the case for graphs of five vertices, we look at the dimension of $M / M^{2}$ over $R / M$. If $M / M^{2}$ is onedimensional, then $R$ is a PIR and $\mathbb{A} \mathbb{G}(R)$ is isomorphic to the graph in Figure 6 . If not, then $M / M^{2}$ must have $R / M$-dimension 2 , as any other possible value for this dimension would require $R$ to have more than eight ideals (as argued in the proof of Theorem 3.3). If $M^{2}=0$, then $\mathbb{A} \mathbb{G}(R) \cong K_{6}$ (this happens, for example, if $R=\mathbb{F}_{4}[x, y] /(x, y)^{2}$, where $\mathbb{F}_{4}$ is a field with 4 elements). We suppose now that $M^{2} \neq 0$. We know that $R$ has exactly four ideals (call them $A, B, C$ and $D$ ) other than $0, M^{2}, M$ and $R$. If all four of these ideals contain $M^{2}$, then $M / M^{2}$ has four one-dimensional subspaces, which means that $R / M \cong \mathbb{F}_{3}$ and that 
every ideal, except for $M$, is principal. Since $\operatorname{ann}\left(M^{2}\right)=M$, we have $\left|M^{2}\right|=R / M=3$ by Lemma 3.2. Therefore, all of the indices in the ideal lattice are 3. This means that each of $A, B, C$ and $D$ has size 9 . Moreover, by Lemma 3.2, each of $\operatorname{ann}(A), \operatorname{ann}(B), \operatorname{ann}(C)$ and $\operatorname{ann}(D)$ also has size 9 . We have three possibilities. One possibility is that each of $A, B, C$ and $D$ is its own annihilator. If this were true, then $\mathbb{A} G(R) \cong K_{1,5}$, which is impossible as we will see in Theorem 3.5. The second possibility is that two of the ideals each annihilate themselves, and that the other two form an annihilator pair. This happens, for example if $R=\mathbb{Z}_{3}[x, y] /\left(x y, x^{2}+y^{2}\right)$ (see Figure 8 ). The final possibility is that the four ideals form two annihilator pairs. This is the case if $R=\mathbb{Z}_{3}[x, y] /\left(x y, x^{2}-y^{2}\right)$ (see Figure 7 ).

The final case is when $A, B, C$ and $D$ do not all contain $M^{2}$; we will show that this is impossible. In this case, by Lemma 2.2, at least three of these, say $A, B$ and $C$, contain $M^{2}$. Suppose, however, that $D$ does not contain $M^{2}$. Since $M / M^{2}$ contains exactly three one-dimensional subspaces, $R / M$ has size 2. Since $D$ does not contain $M^{2}, D$ is minimal, which means, by Lemma 3.2, that $D$ has size 2 . We investigate separately the case when $D$ is contained in $M^{2}$ and the case when it is not.

If $D$ is contained in $M^{2}$, then $M^{2}$ must have size at least 4, which means, by Lemma 3.2, that the index of $\operatorname{ann}\left(M^{2}\right)$ must be at least 4 . Therefore, $\operatorname{ann}\left(M^{2}\right) \neq M$, which means that $D=M^{3}$. Since there are no ideals contained strictly between $M^{2}$ and $D=M^{3}, M^{2} / D$ is a one-dimensional $R / M$-vector space, which implies that the index of $D$ in $M^{2}$ is precisely 2 . Thus, every index in the ideal lattice of $R$ is equal to 2. Note that each of $A, B$ and $C$ is principal. By what we have shown about the indices, each also has size 8. By Lemma 3.2, the annihilator of each has 4 elements, which means that $\operatorname{ann}(A)=\operatorname{ann}(B)=\operatorname{ann}(C)=M^{2}$. But then $0=M^{2}(A+B)=M^{2}(M)=M^{3}$, which is a contradiction.

Suppose, on the other hand, that $D$ is not contained in (nor does it contain) $M^{2}$. Once again, $D$ must be minimal and, as before, $D$ must have size 2 , as must $M^{2}$ (since it is also minimal in this case). The ideal $D+M^{2}$ must have 4 elements. In this case, note that $A, B$ and $C$ are the only four-element ideals of $R$. For concreteness, assume that $D+M^{2}=A$. Then $M A=M D+M\left(M^{2}\right)=0$. Therefore, we may view $A$ as an $R / M$ vector space. However, since $A$ contains exactly two nontrivial $R / M$-subspaces $\left(D\right.$ and $\left.M^{2}\right)$, this is impossible.

In $\left[\mathrm{AAB}^{+} 14\right.$, Theorem 7], the authors establish restrictions on the complete graphs that are realizable as annihilating-ideal graphs of commutative rings. Specifically, they prove that $K_{n}$ is realizable as $\mathbb{A} \mathbb{G}(R)$ only if $n \in\{1,2\}$, or $R$ is a finite local ring with maximal ideal $M$ such that $M^{2}=0$, and

$$
n=\sum_{1 \leq k \leq d}\left(\begin{array}{l}
d \\
k
\end{array}\right)_{q}
$$

where $q$ is the order of $R / M$, and $d$ is the $R / M$-dimension of $M$. Although it is not mentioned explicitly, each of their graphs is, in fact, realizable (take $\mathbb{F}_{q}\left[x_{1}, \ldots, x_{d}\right] /\left(x_{1}, \ldots, x_{d}\right)^{2}$ for suitable $q$ and $d$ ).

In [BR11a, Theorem 2.6], necessary conditions are established for the realization of a star graph as an annihilating-ideal graph. This problem is then studied extensively in [YWG15]. Our next result offers a different proof of [YWG15, Corollary 3.4]. 
Theorem 3.5. The graph $K_{1, n}$ is realizable as $\mathbb{A G}(R)$ for some commutative ring $R$ if and only if $n=1,2$ or $n=2^{k}+2$ for some positive integer $k$.

Proof. The $n=1$ and $n=2$ cases are established in [BR11a, Theorem 2.6]. Suppose now that $R$ is a ring, and that $\mathbb{A} \mathbb{G}(R) \cong K_{1, n}$ for some $n \geq 3$. Again using [BR11a, Theorem 2.6], we see that $R$ must be local with maximal ideal $M$ such that $M^{3}=0$ but $M^{2} \neq 0$ (in particular, $M^{2}$ is minimal) and such that, for any two proper distinct ideals $A$ and $B$, both different from $M^{2}, A B=M^{2}$. Since $n \geq 3$, Lemma 2.1 shows that $M$ is not principal.

We claim, however, that $M$ must be 2 -generated. Suppose, instead, that $x, y$ and $z$ are three elements of $M$ which are linearly independent, $\bmod M^{2}$. Since $M^{2}$ is minimal, we must have $x y=\lambda x z$ for some $\lambda \in R$ (note that neither $x y$ nor $x z$ is zero). But then $x(y-\lambda z)=0$, which is impossible since neither $x$ nor $y-\lambda z$ is contained in $M^{2}$. Therefore, $M$ must be 2-generated.

We next claim that $u^{2}=0$ for every $u \in M$. This is immediate if $u \in M^{2}$, so we assume otherwise. Pick $v$ such that $u$ and $v$ are linearly independent, $\bmod M^{2}$. If $u^{2} \neq 0$, then, following the argument above, we must have $\lambda u^{2}=u v$ for some $\lambda \in R$. But this is impossible, as neither $u$ nor $\lambda u-v$ is in $M^{2}$. Thus, $u^{2}=0$.

We now claim that $|R / M|=2^{k}$ for some positive integer $k$. Since $R / M$ is a finite field, it must be the case that $|R / M|=p^{m}$ for some prime $p$ and some positive integer $m$. Suppose for a contradiction that $p$ is odd. Write $M=(x, y)$ for suitable $x, y \in R$. Since neither $x$ nor $y$ is in $M^{2}$, we know that $x^{2}=0$ and $y^{2}=0$ but $x y \neq 0$. Consider the element $x+\frac{p-1}{2} y$. We have

$$
0=\left(x+\frac{p+1}{2} y\right)^{2}=x^{2}+(p+1) x y+\frac{p^{2}+2 p+1}{4} y^{2}=(p+1) x y .
$$

This contradicts the fact that $x y \neq 0$. Therefore, we must have $|R / M|=2^{k}$ for some $k$. The number of proper nonzero ideals of $R$ is the same as the number of $R / M$-subspaces of $M / M^{2}$, namely $2^{k}+3$ (appealing again to the $q$-binomial coefficient). This means that $n=2^{k}+2$.

On the other hand, we claim that each of these star graphs is, in fact, realizable. Let $k$ be a positive integer, and let $q=2^{k}$. Let $R=\mathbb{F}_{q}[x, y] /\left(x^{2}, y^{2}\right)$. This ring is local, with $M=(x, y), M^{2}=(x y)$ and $2^{k}+3$ proper nonzero ideals. Further, note that, if $\alpha$ is any element of $\mathbb{F}_{q}$, then $(x+\alpha y)^{2}=0$. Therefore, $\operatorname{ann}(y)=(y)$ and $\operatorname{ann}(x+\alpha y)=(x+\alpha y)$ for every $\alpha \in \mathbb{F}_{q}$. Thus, $\mathbb{A} \mathbb{G}(R) \cong K_{1,2^{k}+2}$.

Remark 3.6. Note that, by [BR11b, Theorem 2.3], $K_{m, n}$ is not realizable as an annihilatingideal graph for any $m, n \geq 2$.

In the spirit of the "realization problem", we next offer a simple observation about the possible girths of the graphs which we have been studying. In [BR11a, Theorem 2.1], it is shown that, if $\mathbb{A} \mathbb{G}(R)$ contains a cycle, then $\operatorname{gr}(\mathbb{A} \mathbb{G}(R)) \leq 4$. For commutative rings with finitely many ideals, we can prove a sharper bound.

Theorem 3.7. Let $R$ be a commutative ring with finitely many ideals such that $\mathbb{A} \mathbb{G}(R)$ contains a cycle. Then $\operatorname{gr}(\mathbb{A} \mathbb{G}(R))=3$.

Proof. Suppose first that $R$ is local with maximal ideal $M$. Let $N=\operatorname{ann}(M)$. Note that $N A=0$ for every proper ideal $A$ of $R$. Therefore, if $\mathbb{A} \mathbb{G}(R)$ has a cycle, then it contains a 
pair of ideals $A$ and $B$ such that $A B=0$ and $A, B \neq N$. Then $[A]-[N]-[B]-[A]$ is a cycle of length 3.

We may now assume that $R$ is not local. Then $R=S \times T \times X$ for some local rings $S$ and $T$ and a (possibly trivial) ring $X$. If $X \neq 0$, then $[(R, 0,0)]-[(0, S, 0)]-[(0,0, T)]-[(R, 0,0)]$ is a cycle of length 3. Therefore, we may focus on the case where $R=S \times T$ for local rings $S$ and $T$ with maximal ideals $J$ and $K$, respectively. Note that if $S$ and $T$ are fields, then $\mathbb{A} \mathbb{G}(R) \cong K_{2}$ and is acyclic. If, on the other hand, neither $R$ nor $S$ is a field, then $[(J, 0)]-[(\operatorname{ann}(J), \operatorname{ann}(K))]-[(0, K)]-[(J, 0)]$ is a cycle of length 3 . Assume now that $S$ is a field and that $T$ is not. If $K$ is the only nontrivial proper ideal of $T$, then $\mathbb{A G}(R) \cong P_{4}$ is acyclic. On the other hand, suppose that $T$ has more than one nontrivial proper ideal. Let $A$ be a minimal ideal of $T$, and let $B$ be minimal with respect to not being equal to $A$. Then $A B=0=A^{2}$ since $A, B \subseteq K=\operatorname{ann}(A)$. Then $[(0, A)]-[(0, B)]-[(S, A)]-[(0, A)]$ is cycle of length 3 .

Our next result concerns the relationship between $\mathbb{A} \mathbb{G}(R)$ and $\Gamma_{E}(R)$ (recall that this was defined in [SW11], based on an idea in [Mul02]). Recall that the vertices of $\Gamma_{E}(R)$ are equivalence classes of elements of $R$, under the following equivalence: $x \sim y$ if $\operatorname{ann}(x)=$ $\operatorname{ann}(y)$. Two vertices, $[x]$ and $[y]$, of $\Gamma_{E}(R)$ are connected by an edge if $x y=0$. A ring $R$ is called right p-injective if $\operatorname{ann}_{l}\left(\operatorname{ann}_{r}(a)\right)=R a$ for every $a \in R$ (see, for example, [NY95]). For artinian commutative rings, this is equivalent (by Lemma 2.8) to $R$ being QF. The following is immediate.

Proposition 3.8. Let $R$ be a commutative ring. Let $x, y \in R$. If $(x)=(y)$, then $x \sim y$. The converse holds for every pair $x, y \in R$ precisely when $R$ is p-injective.

Thus, if $R$ is p-injective, there is a natural well-defined injective graph homomorphism $\varphi: \Gamma_{E}(R) \rightarrow \mathbb{A} \mathbb{G}(R)$, given by $\varphi([x])=[(x)]$. In this case, we may view $\Gamma_{E}(R)$ as an (induced) subgraph of $\mathbb{A} \mathbb{G}(R)$.

In the case where $R$ is an artinian PIR, we have an isomorphism.

Theorem 3.9. Let $R$ be a commutative artinian PIR. Then $\Gamma_{E}(R) \cong \mathbb{A} \mathbb{G}(R)$.

Proof. Since $R$ is an artinian commutative PIR, it is immediate that $R$ is QF and, therefore, that the map $\varphi$ given above is well-defined and injective. It suffices to prove that $\varphi$ is surjective. Let $I$ be an ideal of $R$. Since $R$ is a PIR, $I=(a)$ for some $a \in R$. By Proposition 3.8, $[I]=\varphi([a])$. Thus, $\varphi$ is onto, and this establishes the desired isomorphism.

Although the connection that we have drawn between $\Gamma_{E}(R)$ and $\mathbb{A} \mathbb{G}(R)$ is only a first step, we include it in the hope that this connection will motivate further results about both of these structures.

\section{Acknowledgments}

This research was partially supported by a Wellesley College Brachman Hoffman Small Grant. The authors are also extremely grateful to the anonymous referee, whose careful reading and thoughtful comments led to marked improvements. 


\section{References}

$\left[\mathrm{AAB}^{+} 14\right]$ G. Aalipour, S. Akbari, M. Behboodi, R. Nikandish, M. J. Nikmehr, and F. Shaveisi. The classification of the annihilating-ideal graphs of commutative rings. Algebra Colloq., 21(2):249-256, 2014.

[AL99] David F. Anderson and Philip S. Livingston. The zero-divisor graph of a commutative ring. J. Algebra, 217(2):434-447, 1999.

[AM69] M. F. Atiyah and I. G. Macdonald. Introduction to commutative algebra. Addison-Wesley Publishing Co., Reading, Mass.-London-Don Mills, Ont., 1969.

[AM04] S. Akbari and A. Mohammadian. On the zero-divisor graph of a commutative ring. J. Algebra, 274(2):847-855, 2004.

[AM06] S. Akbari and A. Mohammadian. Zero-divisor graphs of non-commutative rings. J. Algebra, 296(2):462-479, 2006.

[AM07a] S. Akbari and A. Mohammadian. On zero-divisor graphs of finite rings. $J$. Algebra, 314(1):168-184, 2007.

[AM07b] David F. Anderson and S. B. Mulay. On the diameter and girth of a zero-divisor graph. J. Pure Appl. Algebra, 210(2):543-550, 2007.

[AMY03] S. Akbari, H. R. Maimani, and S. Yassemi. When a zero-divisor graph is planar or a complete $r$-partite graph. J. Algebra, 270(1):169-180, 2003.

[Bec88] István Beck. Coloring of commutative rings. J. Algebra, 116(1):208-226, 1988.

[BR11a] M. Behboodi and Z. Rakeei. The annihilating-ideal graph of commutative rings I. J. Algebra Appl., 10(4):727-739, 2011.

[BR11b] M. Behboodi and Z. Rakeei. The annihilating-ideal graph of commutative rings II. J. Algebra Appl., 10(4):741-753, 2011.

[CHSW10] Hung-Jen Chiang-Hsieh, Neal O. Smith, and Hsin-Ju Wang. Commutative rings with toroidal zero-divisor graphs. Houston J. Math., 36(1):1-31, 2010.

[CSWSS12] Jim Coykendall, Sean Sather-Wagstaff, Laura Sheppardson, and Sandra Spiroff. On zero divisor graphs. In Progress in commutative algebra 2, pages 241-299. Walter de Gruyter, Berlin, 2012.

[DD05] Frank DeMeyer and Lisa DeMeyer. Zero divisor graphs of semigroups. J. Algebra, 283(1):190-198, 2005.

[Har82] Manabu Harada. Self mini-injective rings. Osaka J. Math., 19(3):587-597, 1982.

[Hun68] Thomas W. Hungerford. On the structure of principal ideal rings. Pacific J. Math., 25:543-547, 1968. 
[LaG08] John D. LaGrange. On realizing zero-divisor graphs. Comm. Algebra, 36(12):4509-4520, 2008.

[LaG16] John D. LaGrange. Annihilators in zero-divisor graphs of semilattices and reduced commutative semigroups. J. Pure Appl. Algebra, 220(8):2955-2968, 2016.

[Mul02] S. B. Mulay. Cycles and symmetries of zero-divisors. Comm. Algebra, 30(7):3533-3558, 2002.

[NY95] W. K. Nicholson and M. F. Yousif. Principally injective rings. J. Algebra, 174(1):77-93, 1995.

[NY97] W. K. Nicholson and M. F. Yousif. Mininjective rings. J. Algebra, 187(2):548578, 1997.

[Red07] Shane P. Redmond. On zero-divisor graphs of small finite commutative rings. Discrete Math., 307(9-10):1155-1166, 2007.

[Smi06] Neal O. Smith. Planar zero-divisor graphs. In Focus on commutative rings research, pages 177-186. Nova Sci. Publ., New York, 2006.

[SW11] Sandra Spiroff and Cameron Wickham. A zero divisor graph determined by equivalence classes of zero divisors. Comm. Algebra, 39(7):2338-2348, 2011.

[Yao08] Yongwei Yao. Infinite rings with planar zero-divisor graphs. Comm. Algebra, 36(11):4068-4077, 2008.

[YWG15] Houyi Yu, Tongsuo Wu, and Weiping Gu. Artinian Local Rings Whose Annihilating-ideal Graphs Are Star Graphs. Algebra Colloq., 22(1):73-82, 2015.

Amanda R. Curtis

Department of Mathematics

University of California, Santa Barbara

Email: acurtis@math.ucsb.edu

Alexander J. Diesl

Department of Mathematics

Wellesley College

Wellesley, MA 02481 USA

Email: adiesl@wellesley.edu

Jane C. Rieck

Email: jane.rieck@gmail.com 